www.jmscr.igmpublication.org

Impact Factor 5.84

Index Copernicus Value: 83.27

ISSN (e)-2347-176x ISSN (p) 2455-0450

crossref DOI: https://dx.doi.org/10.18535/jmscr/v5i7.176

Journal Of Medical Science And Clinical Research

\title{
Application of SEM model in identifying the interrelationship of risk factors of microalbuminuria among type II diabetes mellitus
}

Authors

\begin{abstract}
AJW Felix ${ }^{1}$, A Viknesh Ambayiram², P Kaliyani ${ }^{3}$, PK Govindarajan ${ }^{4}$, E Venmathi ${ }^{5}$
${ }^{1}$ Reader cum Statistician, Department of Community Medicine, Rajah Muthiah Medical College and Hospital, Chidambaram, Tamilnadu, India

${ }^{2}$ Postgraduate, Department of Community Medicine, Rajah Muthiah Medical College and Hospital,

Chidambaram, Tamilnadu, India
\end{abstract}

${ }^{3}$ Reader cum Epidemiologist, Department of Community Medicine, Rajah Muthiah Medical College and Hospital, Chidambaram, Tamilnadu, India

${ }^{4} \mathrm{Head}$ of the Department, Department of Community Medicine, Rajah Muthiah Medical College and Hospital, Chidambaram, Tamilnadu, India

${ }^{5}$ Postgraduate, Department of Community Medicine, Rajah Muthiah Medical College and Hospital,

Chidambaram, Tamilnadu, India

Corresponding Author

Prof .AJW Felix

Email:Amfelix@rediffmail.com, Phoneno: 9443561351

\begin{abstract}
Background: Diabetes is one of the largest health emergencies of the $21^{\text {st }}$ century. In future there will not only be increase in the incidence and prevalence of diabetes but also there will be an increase in economic burden, disability and life years lost due to disease. Microalbuminuria is used as predictor for early micro and macrovascular changes in diabetes.

Objectives: The objectives wereto find the interrelationship between the known risk factors of microalbuminuria among diabetic patients.

Methodology: A descriptive cross sectional study was carried out in the urban field practice area of urban health centre between October 2015 and July 2016, among 200 study participants who had type II diabetes mellitus.

Results: The prevalence of microalbuminuria among the study participants was $41.5 \%$. According to Structural equation modelling, Age, duration of diabetes, duration of hypertension, systolic blood pressure and diastolic blood pressure were found to influence the occurrence of microalbuminuria in type II diabetes mellitus patients.

Conclusion: An intensive education of patients as well as general practitioners on microalbuminuria, risk factors and their interactions were mandatory to prevent various complications.

Keywords: Microalbuminuria, Type II diabetes mellitus, duration of diabetes, duration of hypertension.
\end{abstract}

\section{Introduction}

Global prevalence of diabetes mellitus (DM) has increased dramatically over the past 2 decades (from 177 million in 2000 and 285 million in 2010) and if this situation continues, by 2030 more than 360 million people will have DM. ${ }^{(1)}$ 
expecting that DM remains among the main causes of human mortality and morbidity ${ }^{(1,4)}$. Diabetes mellitus is known for its microvascular and macrovascular complications that contribute to high rates of mortality and morbidity associated with this disease ${ }^{(4)}$ As the number of persons with diabetes increases, the development of microvascular complications like retinopathy, nephropathy and neuropathy also rises. ${ }^{(2)}$ The magnitude of damage caused by these microvascular complications of diabetes stresses the need for sensitive markers of screening for retinopathy and nephropathy ${ }^{(2)}$.

Kidney disease in type $2 \mathrm{DM}$ is more complex for several reasons, mainly because the onset of DM is almost unknown and may be many years before diagnosis. ${ }^{(1)}$ Diabetic nephropathy (DN) is a progressive kidney disease and, at present, in many countries, is the most common cause of endstage renal disease (ESRD). ${ }^{(5,8)}$ The risk of developing DN starts with albuminuria, progressing from micro- to macroalbuminuria. ${ }^{(8)}$ Microalbuminuria associated with diabetes is an independent risk factor for developing diabetic nephropathy ${ }^{(3)}$ also as one of the initial markers of this condition which indicates an increased risk of deteriorating renal function and mortality ${ }^{(5,6,8)}$. With no therapeutic intervention is in place, about $20 \%$ to $40 \%$ of type 2 diabetic patients with microalbuminuria, progress to overt nephropathy and finally approximately $20 \%$ develop end-stage renal failure ${ }^{(1)}$

Microalbuminuria is defined as subclinical urinary excretion of albumin and it has been shown to predict the progression of renal disease in patients with diabetes ${ }^{(6)}$ Microalbuminuria, defined as a urinary albumin excretion rate of 30 to $300 \mathrm{mg} / \mathrm{d}$, or 20 to $200 \mathrm{mg} / \mathrm{min}$, is the earliest clinical sign of nephropathy $^{(9)}$ In diabetics, increased glucose levels leads indirectly, through various metabolic pathways cause endothelial damage including glycocalyx disruption ${ }^{(11)}$ In the capillaries of the renal glomeruli, albumin binds to the glycated basement membrane which leads to increased basement membrane thickening and is a characteristic of diabetic microangiopathy ${ }^{(10)}$
This endothelial damage is reflected by microalbuminuria ${ }^{(11)}$. microalbuminuria is also a marker of greatly increased cardiovascular morbidity and mortality in patients with type I or type II DM. ${ }^{(9)}$ Microalbuminuria represents the simplest and most sensitive prognostic factor to evaluate the risk of overt nephropathy in diabetes, representing the first stage of progressive diabetic renal disease ${ }^{(11)}$ It is at this stage that one can hope to reverse diabetic nephropathy or prevent its progression. $^{(13)}$

Early recognition and treatment of microalbuminuria can prevent irreversible complications such as kidney problems, which ultimately leads to kidney failure and cardiovascular events ${ }^{(1)}$. Though renal biopsy that is the gold standard diagnostic investigation for diabetic nephropathy (1). Microalbuminuria is the most sensitive marker widely used as diagnostic investigation (11). A diagnosis of microalbuminuria can be made by measuring its excretion rate during 24 hours or in an overnight urine collection, or by measuring albumin/creatinine ratio or albumin concentration in the morning or a random urine sample. ${ }^{(13)}$

The Developing Education on Microalbuminuria for Awareness of Renal and Cardiovascular risk in Diabetes (DEMAND), a multinational crosssectional clinic/center-based study, revealed that approximately $50 \%$ of type 2 diabetic patients had micro- or macroalbuminuria, and Asians had a higher prevalence of albuminuria compared with Caucasian patients ${ }^{(8)}$ Patients with type 2 diabetes often have a clustering of risk factors ${ }^{(5)}$ leading to the microvascular complications which are the duration of diabetes mellitus, poor glycaemic control and systolic hypertension ${ }^{(2)}$ The main risk factors identified in DEMAND were HbA1c, systolic blood pressure (BP), ethnicity, retinopathy, duration of diabetes, kidney function, body height, and smoking. ${ }^{(8)}$ Because of the ageing population and an increase in obesity and sedentary lifestyle, the prevalence of diabetes is growing, particularly in Asia. The prevalence of hypertension is even greater in patients with type 2 diabetes and elevated urinary albumin excretion (UAE). ${ }^{(5)}$ systolic blood pressure (BP) is found to 
be an independent risk factor for albuminuria in type 2 diabetes mellitus (T2DM). Based on these studies, the prevalence of albuminuria is expected to be higher in T2DM with hypertension relative to T2DM without hypertension, but it has been largely unexplored. ${ }^{(17)}$

Thus the present study was carried out with objectives of finding the interrelationship between the known risk factors of microalbuminuria among diabetic patients.

\section{Methodology}

The study was a descriptive cross-sectional type conducted in carried out in the field practice area of Urban Health centre (UHC) under division of community medicine, Rajah Muthiah Medical Collge and Hospital, Chidambaram, Tamilnadu. The study was carried out for a period of 10 months from October 2015 to July 2016. People living in field practice area of urban health centre, aged more than 30 years and had type II diabetes mellitus had been included in the study. The study was ethically approved by the ethical committee of the same institute. Also informed consent was obtained from participants prior to interview.

\section{Sample Size Calculation}

With absolute prevalence fixed as $7 \%$ and prevalence as 0.4 the sample size was calculated to be 188 which was then rounded upto 200 thus taken as the sample size. Convenient sampling was applied by house to house visit in the study area till the required sample size was achieved. Persons aged $>30$, non diabetic, sick, pregnant and rheumatic heart diseases were excluded.

\section{Study tool and data collection}

Study tool used in the study was a pretested semi structured questionnaire including details on socio-demographic details, anthropometry, clinical examination and biochemical examination. Proforma was prepared in English and local dialect, was used during interview to make it convenient for the participants.

In the study tool, Socio-demographic details included age, sex, marital status, educational status and income. The information regarding diabetes and hypertension were collected which included details on the duration of diabetes and hypertension, whether they were taking their antidiabetic and anti-hypertensive medications regularly and the drugs that they were taking to treat the conditions. Further information was collected on lifestyle habits- smoking and alcohol consumption.

Following the data collection, height and weight of the study participants were measured. body Mass Index (BMI) was calculated by using the formula weight $(\mathrm{kg}) /$ height $(\mathrm{m}) 2$. Blood Pressure was measured by sphygmomanometer and Stethoscope in sitting position. Urine samples of the participants were collected in sterile containers and were sent to the laboratory within 20 minutes for the estimation of urine albumin and urine creatinine values.

\section{Data Analysis}

Data collected was entered in Microsoft 2013 excel spread sheet, compiled and analysed using IBM SPSS Version 21 statistical package. Descriptive statistics, Pearson chi-square test and logistic regression analysis were performed to find out association between microalbuminuria and selected risk factors, co-morbid conditions.

\section{Results}

Out of the 200 study participants with type II diabetes mellitus, $59.5 \%$ belonged to the age group of 51-70 years. The mean age of the study participants was $57.34 \pm 10.87$ years. (Table 1)

$42.5 \%$ of the study participants had systolic blood pressure of more than $140 \mathrm{mmHg}$ and $34 \%$ had their systolic blood pressure between 140 and 159 $\mathrm{mmHg}$. Diastolic blood pressure was recorded to be more than $90 \mathrm{mmHg}$ in $47 \%$ of the study participants and between 80 to $89 \mathrm{mmHg}$ in $29 \%$ of the study participants (Table: 2). The duration of diabetes mellitus among $50.5 \%$ of the study participants was 2 to 5 years. Among the study participants, $43 \%$ had hypertension for 2 to 5 years. (Table: 3 ) 
According to Structural equation modelling, Age, duration of diabetes, duration of hypertension, systolic blood pressure and diastolic blood pressure were found to influence the occurrence of microalbuminuria in type II diabetes mellitus patients. All these factors were also found to be influencing one another, age was found to influencing duration of diabetes mellitus. Both age and duration of diabetes were found to be influencing systolic blood pressure and all the three were found to be influencing microalbuminuria. This interaction extents to almost all the selected risk factors. (fig 1)

Table: 1 Distribution of study participants according to age.

\begin{tabular}{|l|c|c|}
\hline Age & $\mathrm{N}$ & $\%$ \\
\hline $31-40$ & 19 & 9.5 \\
\hline $41-50$ & 38 & 19 \\
\hline $51-60$ & 67 & 33.5 \\
\hline $61-70$ & 52 & 26 \\
\hline$>70$ & 24 & 12 \\
\hline Total & 200 & 100 \\
\hline
\end{tabular}

Table: 2 Distribution of study participants according to systolic and diastolic blood pressure.

\begin{tabular}{|l|c|c|}
\hline Systolic blood pressure $(\mathrm{mm} / \mathrm{Hg})$ & $\mathrm{N}$ & $\%$ \\
\hline$<120$ & 47 & 23.5 \\
\hline $120-139$ & 68 & 34 \\
\hline $140-159$ & 77 & 38.5 \\
\hline$\geq 160$ & 8 & 4 \\
\hline Diastolic blood pressure $(\mathrm{mm} / \mathrm{Hg})$ & $\mathrm{N}$ & $\%$ \\
\hline$<80$ & 48 & 24 \\
\hline $80-89$ & 58 & 29 \\
\hline $90-99$ & 74 & 37 \\
\hline$\geq 100$ & 20 & 10 \\
\hline Total & 200 & 100 \\
\hline
\end{tabular}

Table: 3 Distribution of study participants according to the duration of diabetes mellitus and hypertension.

\begin{tabular}{|l|c|c|}
\hline Duration of diabetes mellitus. (years) & $\mathrm{N}$ & $\%$ \\
\hline$\leq 1$ & 33 & 16.5 \\
\hline $2-5$ & 101 & 50.5 \\
\hline $6-10$ & 32 & 16 \\
\hline $11-20$ & 17 & 8.5 \\
\hline$>20$ & 17 & 8.5 \\
\hline Duration of hypertension (years) & $\mathrm{N}$ & $\%$ \\
\hline$\leq 1$ & 19 & 19 \\
\hline $2-5$ & 43 & 43 \\
\hline $5-10$ & 19 & 19 \\
\hline $10-20$ & 17 & 17 \\
\hline$>20$ & 2 & 2 \\
\hline Total & 100 & 100 \\
\hline
\end{tabular}

Fig: 1 Relationship between selected risk factors and microalbuminuria using structural equation modelling.

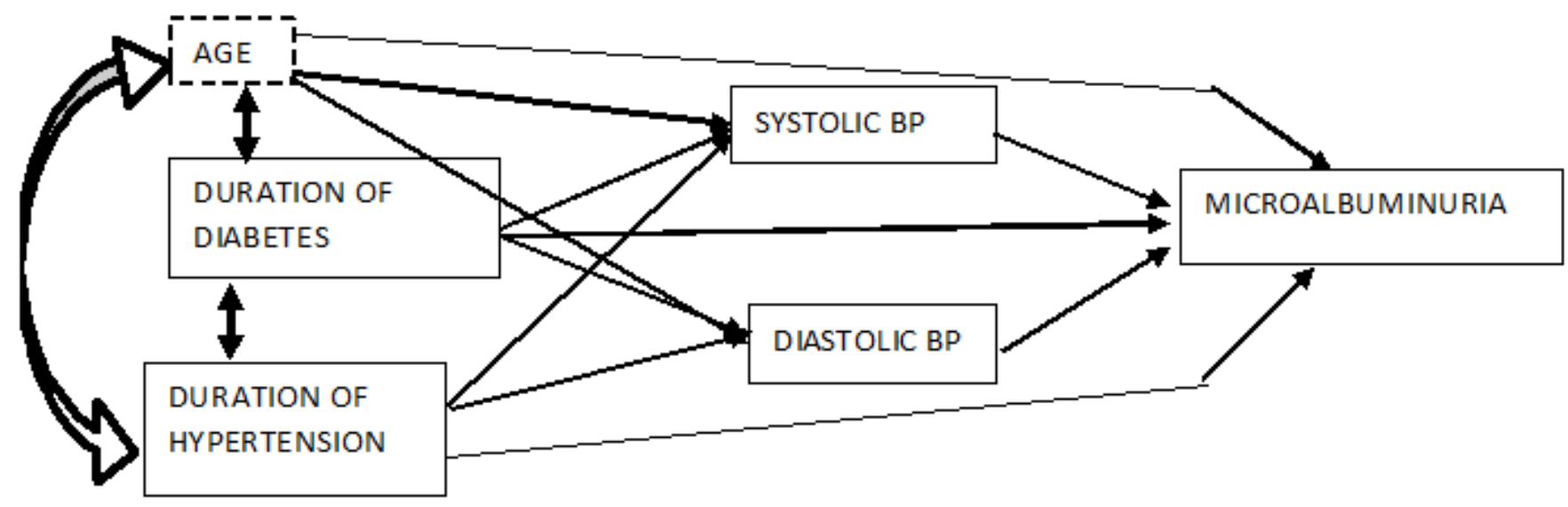

\section{Discussion}

Microalbuminuria is not only the earliest manifestation of diabetic nephropathy but is also a significant risk factor for the progression of nephropathy in diabetic patients ${ }^{(17)}$ As one of the first clinical symptoms of diabetic nephropathy it may progress to macroalbuminuria and the progressive loss of glomerular filtration rate (GFR) and finally the end stage renal disease. Elder patients often suffer from other diseases, 
including hypertension and atherosclerosis, which can also damage the renal parenchyma. ${ }^{(1,5)}$

Microalbuminuria is thus an indicator of possible vascular disease requiring aggressive intervention to reduce cardiovascular risk. Various epidemiological and cross-sectional studies have reported marked variation in the prevalence of microalbuminuria, ranging from less than $10 \%$ in the United Kingdom8,9 to $18 \%$ in Singapore 10 and more than $30 \%$ in southern India. (9) Microalbuminuria is likely to be found in onethird or more of diabetic patients ${ }^{(1)}$

\section{Prevalence}

In the present study the prevalence of microalbuminuria among the type 2 diabetes mellitus patients in urban Chidambaram was found to be $41 \%$. This is similar to the result of studies conducted in neighbouring Asian countries reported different prevalence rates of microalbuminuria ranging from $14.2 \%$ in Iran to $36.3 \%$ in India. While in other European countries, the total prevalence was observed as 47\%. ${ }^{(1)}$ Also similar findings were reported by Aldani et al and Chota et al ${ }^{(8,13)}$

According to a report by the World Health Organization (WHO), the prevalence rates of nephropathy after 15 years of diabetes ranged between 17.7 and $56.6 \%$ in men and between 11.9 and $71 \%$ in women ${ }^{(2)}$ supporting the outcome of our result. It was also observed that the prevalence of microalbuminuria was found to be $52.04 \%$ among all diabetic patients ${ }^{(10)}$.

The MAPS is the first large multicentre epidemiological study conducted in Asia to determine the prevalence of microalbuminuria and macroalbuminuria in patients with type 2 diabetes and hypertension. The prevalence of microalbuminuria was slightly higher than the rates of $17 \%$ to $21 \%$ reported from western population-based studies in patients with diabetes. ${ }^{(5)}$ which is contrast to our study results.

In Zakerkish et al study done in Iran the prevalence rates of microalbuminuria in diabetic patients vary from $16.7 \%$ to $35.2 \%$. In another study carried out by this group in 2008, they showed $33 \%$ microalbuminuria which is much lesser than our results ${ }^{(1)}$ though it shows the increase in the prevalence still lesser than the present study result. In rani et al the prevalence of microalbuminuria was 191 out of $1166,16.4 \%$ in individuals with known diabetes, It means that every 6th individual in the population ofindividuals with diabetes had albuminuria. ${ }^{(2)}$

\section{Age and microalbuminuria}

Age is found to influence occurrence of microalbuminuria. Rani PK et al stated that microalbuminuria occurrence is influenced by age, raised blood pressure and poor glycaemic control (2). Chowta NK et al reported a similar relationship between age and microalbuminuria occurrence in type II diabetes mellitus patients. ${ }^{(13)}$. Similar association with age were also recorded byParving et al ${ }^{(16)}$, Tam TKW et al., in $2004^{(9)}$.

\section{Duration of diabetesand microalbuminuria}

As the duration of diabetes mellitus increased so do the probability being microalbuminuric. Similar association was also reported by Rani PK et al ${ }^{(2)}$. Zakkerkrish $\mathrm{M}$ et al also stated that similar association in their univariate analysis ${ }^{(1)}$. Chowta et al in their study has stated a similar correlation between microalbuminuria and type II diabetes mellitus. Nakhjavani and colleagues showed that only the duration of DM, HDLC, and $\mathrm{Hb}$ A1c were the independent risk factors ${ }^{(1)}$. Tam TKW et al, Agarwal $\mathrm{J}$ et al and Baig et al also stated a similar increase in probability ${ }^{(9,10,12)}$.

\section{Duration of hypertensionand microalbum- inuria}

As the duration of hypertension increased so did the occurrence of microalbuminuria. Similar significant association between duration of hypertension and microalbuminuria in type II diabtes mellitus individuals were reported by AlRubeaan $\mathrm{K}$ et al ${ }^{(4)}$. Microalbuminuria is also characterized by increased prevalence of arterial hypertension ${ }^{(13)}$. Rani $\mathrm{Pk}$ et al also reported similar association. 


\section{Systolic blood pressure and microalbuminuria}

Increase in systolic blood pressure was associated with increase in microalbuminuria incidence. Yang $\mathrm{C}$ et al also reported similar associations between systolic blood pressure and microalbuminuria. Elevated blood pressure is documented as the most significant contributing factor in the pathogenesis and the progressionof abnormal AER and eventually development of diabetic nephropathy in both Type 1 and Type 2 diabetic patients. Type 2 patients with abnormal AER had statistically significant higher SBP and DBP than those with normal AER, $(\mathrm{p}<0.0001)$ (15). Al-Adsani A reported hypertension as independent risk factor associated with albuminuria $^{(8)}$.

\section{Diastolic blood pressure and microalbuminuria}

Increase in diastolic blood pressure was associated with increased occurrence of microalbuminuria. Rani PK et al reported that the diastolic blood pressures were higher in those individualswith macro - or microalbuminuria compared to those with normoalbuminuric $(83.8 \pm 12.9$ vs. $84.0 \pm$ 11.5 vs.81.5 $\pm 11.2, \mathrm{p}=005$ ) For microalbuminuria, the significant variables included increase in age per year Odds Ratio (OR) 1.01 (95\% CI: 1.00-1.04), increase in systolic blood pressure per $\mathrm{mm}$ of $\mathrm{Hg}$ OR 1.01 (95\% CI: 1.00-1.02) ${ }^{(2)}$. Similar findings were also reported by Unni Krishnan et al ${ }^{(2)}$.

\section{Conclusion}

The selected risk factors were found to interact with each other and also with microalbuminuria. An intensive education of patients as well as general practitioners on microalbuminuria, risk factors and their interactions were mandatory to prevent various complications.

\section{Limitations}

The definition of albuminuria and diabetic nephropathy is another source of discrepancy, as well as the methods of measurement of albuminuria and urine collection. The results of the study cannot be generalized because the study was confined to urban population of the field practice area. Recall bias can be present in association with certain variables of the study, where the study participants had to remember past dates. Microalbuminuria has a high biological variability. Though albumin creatinine ratio was used to estimate microalbuminuria in order to overcome this variability, it will not account to the variation fully.

\section{References}

1. Zakkerkish M, Shahbazian HB, Shahbazian H, Latifi SM, MoravejAleali A. Albuminuria and its correlates in type 2 diabetic patients. Iran $\mathbf{J}$ Kidney Dis.2013;7(4):268-76.

2. Rani PK, Raman R, Gupta A, Pal SS, Kulothungan V, Sharma T. Albuminuria and Diabetic Retinopathy in Type 2 Diabetes Mellitus SankaraNethralaya Diabetic Retinopathy epidemiology And Molecular Genetic Study (SNDREAMS, report 12). DiabetolMetabSyndr [Internet]. BioMed Central Ltd;2011;3(1):9. Available from: http://www.dmsjournal.com/content/3/1/9

3. Dwyer JP, Parving H-H, Hunsicker LG, Ravid M, Remuzzi G, Lewis JB. Renal Dysfunction in the Presence of Normoalbuminuria in Type 2 Diabetes: Results from the DEMAND Study. Cardiorenal Med. 2012;2(1):1-10.

4. Al-Rubeaan $\mathrm{K}$, Abu El-Asrar AM, Youssef AM, Subhani SN, Ahmad NA, Al-Sharqawi AH, et al. Diabetic retinopathy and its risk factors in a society with a type 2 diabetes epidemic: A Saudi National Diabetes Registry-based study. Acta Ophthalmol. 2015;93(2):e140-7.

5. Yeung VTF, Lee KF, Chan SH, Wong HY. MicroAlbuminuria Prevalence Study ( MAPS ) in hypertensive type 2 diabetic patients in Hong Kong. 2006;12(3).

6. Liangpunsakul and Chalasani: Relationship between hepatitis $\mathrm{C}$ and microalbuminuria 
7. Manaviat MR, Afkhami M, Shoja MR. Retinopathy and microalbuminuria in type II diabetic patients. 2004;4:1-4.

8. Al-adsani A. Renal Data from the Arab World Risk Factors Associated with Albuminuria in Kuwaiti Adults. 2012;23(4):860-5.

9. Tam TKW, Cheng LPK. The prevalence ofmicroalbuminuria among patients with type II diabetes mellitus in a primary caresetting : cross-sectional study. 2004;10(5):307-11.

10. Aggarwal J, Kumar M. Prevalence of microalbuminuria among rural northIndian population with diabetes mellitus and its correlation with glycosylatedhaemoglobin and smoking. J Clin Diagnostic Res. 2014;8(7):20-2.

11. Jia W, Gao X, Pang C, Hou X, Bao Y, Liu $\mathrm{W}$, et al. Prevalence and risk factors of albuminuria and chronic kidney disease in Chinese population with type 2 diabetes and impaired glucose regulation: Shanghai diabetic complications study ( SHDCS ). 2009;(July):3724-31.

12. Jawed AltafBaig, NailaAsif, Aqiba Sarfaraz; Correlation of Microalbuminuria with Glycosylated Hemoglobin (Hbalc) and Duration of Type 2 Diabetes Mellitus (T2DM) in Male and Female Patients; Middle-East Journal of Scientific Research 24 (9): 2900-2903, 2016.

13. Chowta NK., Pant P, Chowta MN. Microalbuminuria in diabetes mellitus: Association with age, sex, weight and creatinine clearance. Indian J Nephrol.2009;19(2):53-6.

14. Hall V, Thomsen R, Henriksen O, Lohse N. Diabetes in Sub Saharan Africa 19992011: Epidemiology and public health implications. A systematic review. BMC Public Health [Internet]. BioMed Central Ltd; 2011;11(1):564.
15. Lutale JJK, Thordarson H, Abbas ZG, Vetvik K. Microalbuminuria among Type 1 and Type 2 diabetic patients of African origin in Dar EsSalaam, Tanzania. 2007;8:1-8.

16. Parving H-H, Lewis JB, Ravid M, Remuzzi G, Hunsicker LG. Prevalence andrisk factors for microalbuminuria in a referred cohort of type II diabetic patients:a global perspective. Kidney Int. 2006;69(11):2057-63.

17. Yang C, Park JT, Kim YS, Kim YL, Lee $\mathrm{Y}$, Oh Y, et al. Prevalence of diabeticnephropathy in primary care type 2 diabetic patients with hypertension: datafrom the Korean Epidemiology Study on Hypertension III (KEY III study). 2011;(March):3249-55. 